\title{
Feasibility of Replacing the Range Doppler Equation of Spaceborne Synthetic Aperture Radar Considering Atmospheric Propagation Delay with a Rational Polynomial Coefficient Model
}

\author{
Shasha Hou ${ }^{1,2}$, Yuancheng Huang ${ }^{1}$, Guo Zhang ${ }^{2, *}$, Ruishan Zhao ${ }^{3}$ and Peng Jia ${ }^{4}$ \\ 1 School of surveying and Mapping Science and Technology, Xi'an University of Science and Technology, \\ Xi'an 710054, China; 18210063041@stu.xust.edu.cn (S.H.); yuanchenghuang@xust.edu.cn (Y.H.) \\ 2 State Key Laboratory of Information Engineering in Surveying Mapping and Remote Sensing, \\ Wuhan University, Wuhan 430079, China \\ 3 School of Geomatics, Liaoning Technical University, Fuxin 123000, China; zhaoruishan@lntu.edu.cn \\ 4 China Satellite Navigation Office, Beijing 100034, China; jiap@beidou.gov.cn \\ * Correspondence: guozhang@whu.edu.cn; Tel.: +86-139-0718-2592
}

Received: 20 November 2019; Accepted: 17 January 2020; Published: 19 January 2020

\begin{abstract}
Usually, the rational polynomial coefficient (RPC) model of spaceborne synthetic aperture radar (SAR) is fitted by the original range Doppler (RD) model. However, the radar signal is affected by two-way atmospheric delay, which causes measurement error in the slant range term of the RD model. In this paper, two atmospheric delay correction methods are proposed for use in terrain-independent RPC fitting: single-scene SAR imaging with a unique atmospheric delay correction parameter (plan 1) and single-scene SAR imaging with spatially varying atmospheric delay correction parameters (plan 2). The feasibility of the two methods was verified by conducting fitting experiments and geometric positioning accuracy verification of the RPC model. The experiments for the GF-3 satellite were performed by using global meteorological data, a global digital elevation model, and ground control data from several regions in China. The experimental results show that it is feasible to use plan 1 or plan 2 to correct the atmospheric delay error, no matter whether in plain, mountainous, or plateau areas. Moreover, the geometric positioning accuracy of the RPC model after correcting the atmospheric delay was improved to better than $3 \mathrm{~m}$. This is of great significance for the efficient and high-precision geometric processing of spaceborne SAR images.
\end{abstract}

Keywords: synthetic aperture radar; GF-3 satellite; rational polynomial coefficient; range Doppler model; atmospheric propagation delay correction; fitting accuracy; ground control point; geometric positioning accuracy

\section{Introduction}

Since 1999, for the IKONOS satellite, considering technical confidentiality and other factors, American space imaging companies have started to provide rational polynomial coefficient (RPC) models instead of rigorous geometry models to end users as basic imaging products [1]. The RPC model is a universal geometric model of remote sensing satellite sensors, which is usually fitted by a range Doppler (RD) model in spaceborne synthetic aperture radar (SAR) imaging systems. The difference between the RPC and RD models is that the former is a purely mathematical model, is independent of the sensor, and involves simple calculations. Furthermore, with the emergence of various imaging sensors, it is difficult for end users to add new sensor models to existing software systems to process new sensor data, while the RPC model solves this difficulty well. Moreover, from the perspective of 
the multi-source sensor data application, the RPC model also provides a unified geometric model for the joint adjustment of multi-source, high-resolution remote sensing images. In view of these characteristics and factors, the RPC model has been widely used in photogrammetric processing of remote sensing satellite images.

Many scholars have conducted extensive research on the calculations involved in and application of RPC models. Firstly, the direct and iterative least-squares results of the RPC parameters were derived, and terrain-dependent and terrain-independent solution methods were proposed in a photogrammetric system [1,2]. Subsequently, the terrain-independent RPC solution algorithm was studied in detail, which is based on a global digital elevation model (DEM) to interpolate the maximum and minimum elevation of the study area and does not require initial values. It was noted that the third-order RPC model with unequal denominators achieved the highest replacement accuracy [3].

The replacement accuracy of the third-order RPC model was verified for different remote sensing satellite images, whether it is a high-resolution SAR satellite, such as TerraSAR-X, COSMO-SkyMed, and Radarsat-2; or medium-resolution and low-resolution SAR satellites, such as ERS, ALOS, JERS, and ASAR, it achieved accuracies of better than 0.015 pixels [3-5]. In addition, many scholars have used control data and compensation models (such as translation, affine transformation, and polynomial models) to improve the geometric positioning accuracies of RPC models. Consequently, the geometric positioning accuracy of the GF-3 satellite is better than 2 pixels, that of the YaoGan-18 satellite is better than $25 \mathrm{~m}$, and Radarsat can achieve a positioning accuracy of $36.735 \mathrm{~m}$ [6-9].

At present, although the RPC model performs well in terms of substitution and positioning accuracy, the slant range measurement error caused by the two-way atmospheric propagation delay of the SAR signal is still not taken into account in the RPC model, and research has shown that the slant range measurement error will bring the positioning error in the range direction [10]. Meanwhile, some researchers pointed out that the range accuracy of SAR using modern satellites has been verified in centimeter-range after correcting geodynamic, systematic errors, and atmospheric effects [11,12]. Moreover, with the continuous launch of high-resolution SAR satellites, the accuracy and efficiency requirements of SAR image geometry processing have become more stringent. Therefore, in this study, we developed an RPC fitting method that corrects the atmospheric propagation delay, analyzed the feasibility of replacing the RD equation considering atmospheric delay correction with the RPC model, and verified the positioning accuracy of the RPC model of spaceborne synthetic aperture radar (SAR) considering atmospheric propagation delay.

GF-3 is the first C-band multi-polarized SAR satellite with $1 \mathrm{~m}$ resolution in China. It has 12 imaging modes, including SpotLight, StripMap, and ScanSAR, along with a wave imaging mode, and can provide data support services for research on water disaster monitoring and assessment, climate change, oceans, agriculture, forestry, and earthquakes. In this study, a series of experiments was conducted using GF-3 SAR satellite data and manually measured real ground control point (GCP) data from several regions in China.

Based on the RD model, this paper describes a method of fitting the RPC model of a spaceborne SAR satellite considering the atmospheric propagation delay and presents an evaluation of the feasibility of this replacement. Section 2 describes three basic models: the atmospheric propagation delay correction model, RD model, and RPC model. The RPC model fitting and accuracy verification methods that corrected the atmospheric propagation delay are presented in Section 3. The atmospheric propagation delay analysis results and RPC model fitting and positioning accuracy verification are discussed in Section 4. Finally, Section 5 presents the conclusions.

\section{Models}

\subsection{Atmospheric Propagation Path Delay Equation}

The atmospheric propagation path delay of a radar signal $\Delta L_{\text {delay }}$ depends on a complex atmospheric spatial distribution pattern, generally at a specific height $h=h_{t}$, and is represented as 
the integral of the sum of the dry, wet, and ionosphere components of the refractivity $N$, from $h_{t}$ to the top of the atmosphere $h_{\infty}[13,14]$ :

$$
\begin{gathered}
N=\left(k_{1} \frac{P}{T}\right)_{d r y}+\left(k_{2} \frac{e}{T}+k_{3} \frac{e}{T^{2}}+k_{4} W_{\text {cloud }}\right)_{\text {wet }}+\left(k_{5} \frac{n_{e}}{f^{2}}\right)_{\text {ionosphere }}=N_{\text {dry }}+N_{\text {wet }}+N_{\text {ionosphere }} \\
\Delta L_{\text {delay }}=\frac{10^{-6}}{\cos \theta} \int_{h_{t}}^{h_{\infty}}\left(N_{\text {dry }}+N_{\text {wet }}+N_{\text {ionosphere }}\right) d h
\end{gathered}
$$

where $P$ is the total pressure (mbar), $T$ is the temperature $(\mathrm{K}), e$ is the partial pressure of water vapor (mbar), and $W_{\text {cloud }}$ is the cloud water content $\left(\mathrm{g} / \mathrm{m}^{3}\right)$. These meteorological data can be downloaded from the U.S. National Center for Environmental Prediction (NCEP) website (https: //rda.ucar.edu/datasets/ds083.2/). In addition, $n_{e}$ is the electronic density of the ionosphere, which can be obtained by bilinear interpolation in time and space based on the global ionospheric map provided by the European Center for Orbit Determination (CODE) (ftp://cddis.gsfc.nasa.gov/gnss/products/ionex/). Here, $f$ and $\theta$ are the frequency and incidence angle of the radar, respectively, which can be obtained from the auxiliary file of the SAR satellite image. The constant coefficients have the following values: $k_{1}=77.6 \mathrm{~K} / \mathrm{mbar}, k_{2}=26.0 \mathrm{~K} / \mathrm{mbar}, k_{3}=3.75310^{5} \mathrm{~K}^{2} / \mathrm{mbar}, k_{4}=1.45 \mathrm{~m}^{3} / \mathrm{g}$, and $k_{5}=24.03310^{7} \mathrm{~m}^{3} / \mathrm{s}^{2}[15-17]$.

\subsection{Spaceborne SAR RD Equation}

The RD equation establishes the correspondence between the image and object point coordinates from the perspective of the spaceborne SAR imaging geometry. It consists of an earth model equation, SAR doppler equation, and SAR range equation [18]:

$$
\left\{\begin{array}{l}
\frac{X_{t}^{2}+Y_{t}^{2}}{A^{2}}+\frac{Z_{t}^{2}}{B^{2}}=1 \\
f_{D}=-\frac{2}{\lambda R}\left(\vec{R}_{s}-\vec{R}_{t}\right) \cdot\left(\vec{V}_{s}-\vec{V}_{t}\right) \\
R=\sqrt{\left(\vec{R}_{s}-\vec{R}_{t}\right) \cdot\left(\vec{R}_{s}-\vec{R}_{t}\right)}
\end{array}\right.
$$

where $R_{s}$ and $\vec{R}_{t}=\left[X_{t} Y_{t} Z_{t}\right]$ are the position vectors of the SAR satellite and target, respectively; $\vec{V}_{S}$ and $\vec{V}_{t}$ are the satellite and target velocity vectors, respectively; $R$ is the slant range; $\lambda$ is the radar wavelength; $A=6378.137 \mathrm{~km}$ is the mean equatorial radius; and $B=(1-1 / f) A$ is the polar radius with a flattening factor $f=298.255$.

Considering the slant range measurement error caused by the atmospheric propagation delay, the slant range can be expressed as

$$
R=\sqrt{\left(\vec{R}_{s}-\vec{R}_{t}\right) \cdot\left(\vec{R}_{s}-\vec{R}_{t}\right)}+\Delta L_{\text {delay }}
$$

\subsection{RPC Model}

The RPC model uses the ratio polynomial to represent the correspondence between the ground coordinate $D$ (Latitude, Longitude, Height) (the polar stereographic coordinate system is more applicable at 70-75 degrees latitude north or south) and the pixel coordinates $d$ (line, sample) [19]:

$$
\left.\begin{array}{l}
Y=\frac{N_{l}(P, L, H)}{D_{l}(P, L, H)} \\
X=\frac{N_{S}(P, L, H)}{D_{S}(P, L, H)}
\end{array}\right\},
$$


where $N_{l}(P, L, H), N_{s}(P, L, H), D_{l}(P, L, H)$, and $D_{s}(P, L, H)$ are all third-order polynomials. These functions have the form

$$
\begin{aligned}
F(P, L, H)= & a_{1}+a_{2} L+a_{3} P+a_{4} H+a_{5} L P+a_{6} L H+a_{7} P H+a_{8} L^{2}+a_{9} P^{2}+a_{10} H^{2}+a_{11} P L H \\
& +a_{12} L^{3}+a_{13} L P^{2}+a_{14} L H^{2}+a_{15} L^{2} P+a_{16} P^{3}+a_{17} P H^{2}+a_{18} L^{2} H+a_{19} P^{2} H+a_{20} H^{3}
\end{aligned}
$$

where $a_{i}$ is the RPC model coefficient, $i=1,2, \ldots, 20$; Generally, the first parameter coefficient of the denominator term is set to 1 , so there are 78 parameters for RPC model.

Here, $(P, L, H)$ and $(X, Y)$ are the normalized ground and image coordinates, respectively. These coordinates can be expressed as

$$
\left.\begin{array}{c}
P=\frac{D_{\text {lat }}-D_{\text {lat_off }}}{D_{\text {lat_scale }}} \\
L=\frac{D_{\text {lon }}-D_{\text {lon_off }}}{D_{\text {lon_scale }}} \\
H=\frac{D_{\text {hei }}-D_{\text {hei_off }}}{D_{\text {hei_scale }}}
\end{array}\right\}
$$

where $D_{i}(i=$ lat_off, lat_scale, lon_off,lon_scale, hei_off, hei_scale $)$ is the normalized parameter of the ground coordinates and $s_{j}, l_{j}(j=o f f$, scale $)$ are the normalized parameters of the image coordinates.

The RPC fitting results obtained based on a variety of spaceborne SAR sensors have shown that RPC models with third-order and unequal denominators have the highest replacement accuracies [7]. The following experiments were based on this model.

\section{RPC Model Fitting and Accuracy Verification Methods}

The RPC model is essentially a mathematical model. In this study, we used a terrain-independent solution algorithm to calculate the RPC parameters. The core of the algorithm involves establishing a virtual control grid based on the SAR image, DEM data of the coverage area, and forward transformation of the RD model. Then, the RPC parameters are fitted according to the least squares fitting method and the correspondence between the ground coordinates and image pixel coordinates of the virtual control points. However, every certain virtual grid point in space is affected by atmospheric propagation delay, which causes slant range measurement error. Therefore, we used two atmospheric delay correction schemes to correct the slant range values at all virtual grid points, then re-fitted the RPC model and evaluated the fitting and positioning accuracies.

The steps of the algorithm are listed in Figure 1. 


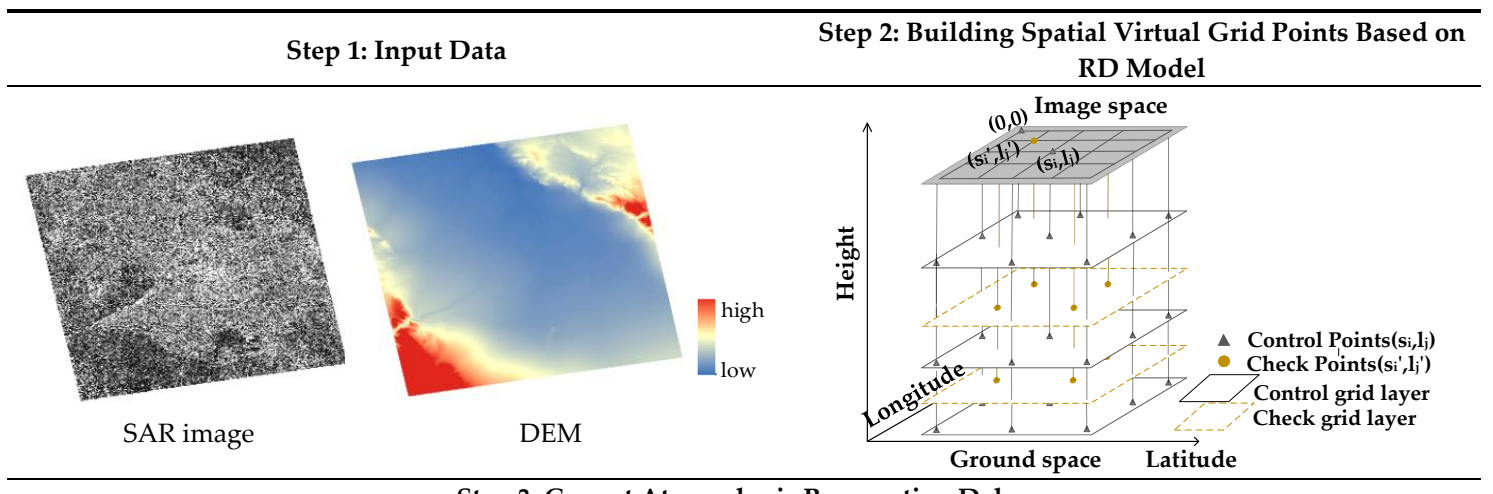

Step 3: Correct Atmospheric Propagation Delay

Plan 1: The atmospheric delay correction value at the point in the center of the scene and the average elevation of the coverage area is used to correct the atmospheric propagation delay at all virtual points.

Plan 2: The atmospheric delay correction value at each virtual grid point is used to correct its own atmospheric delay. Input:

SAR auxiliary file Imaging time, Image size...

Virtual points file Image coordinates(sample, line), Ground coordinates(latitude, longitude), height

NCEP

Meteorological data stored by latitude and longitude grid points $\left(1^{\circ} \times 1^{\circ}\right)$

CODE

Global Ionosphere Map stored by latitude and longitude grid points $\left(5^{\circ} \times 2.5^{\circ}\right)$

Plan 1: Atmospheric delay correction value at the center point

Plan 2: Atmospheric delay correction values at all virtual points

\begin{tabular}{|c|c|c|}
\hline \multicolumn{3}{|c|}{ Step 4: RPC model parameter calculation and accuracy evaluation } \\
\hline $\begin{array}{r}R D_{0} \text { : Original RD mode } \\
\mathrm{RD}_{1} \text { : Original RD model + } 1 \\
\mathrm{RD}_{2} \text { : Original } \mathrm{RD} \text { model }+\mathrm{I}\end{array}$ & \multicolumn{2}{|c|}{$\begin{array}{c}\text { SAR image size }(\text { range } \times \text { azimuth, pixels) }: \mathrm{m} \times \mathrm{n} \\
\text { Plane grid size }(\text { range } \times \text { azimuth, pixels): } \mathrm{x} \times \mathrm{x} \\
\text { Number of elevation layers: } \mathrm{k}\end{array}$} \\
\hline \multicolumn{3}{|c|}{$\begin{array}{l}\text { (1) RPC model fitting } \\
\text { Number of virtual control points: } S=(m / x+1) \times(n / x+1) \times k\end{array}$} \\
\hline 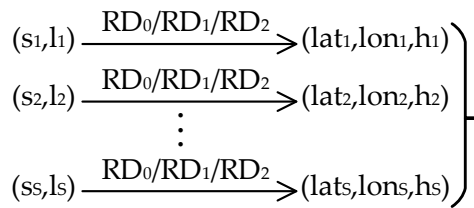 & $\left.\begin{array}{cc}\begin{array}{c}\text { Formulas } \\
(7) \text { and }(8)\end{array} & \mathrm{X}_{1}, \mathrm{Y}_{1}, \mathrm{P}_{1}, \mathrm{~L}_{1}, \mathrm{H}_{1} \\
& \mathrm{X}_{2,}, \mathrm{Y}_{2}, \mathrm{P}_{2}, \mathrm{~L}_{2}, \mathrm{H}_{2} \\
& \\
& \mathrm{X}_{\mathrm{s}}, \mathrm{Y}_{\mathrm{s}}, \mathrm{P}_{\mathrm{s}}, \mathrm{L}_{\mathrm{L}}, \mathrm{H}_{\mathrm{s}}\end{array}\right)$ & $\stackrel{\begin{array}{c}\text { Formulas (5) and (6) } \\
\text { Least squares fitting }\end{array}}{\longrightarrow} \begin{array}{c}\text { Output : } \\
\mathbf{R P C}_{0} / \mathbf{R P C}_{1} / \mathbf{R P C}_{2} \\
\mathbf{M}_{a_{i}}, b_{i}, c_{i}, d_{i}(\mathrm{i}=1,2 \ldots 20)\end{array}$ \\
\hline
\end{tabular}

(2) RPC model fitting accuracy evaluation

Number of virtual check points: $S^{\prime}=(m / x) \times(n / x) \times(k-1)$

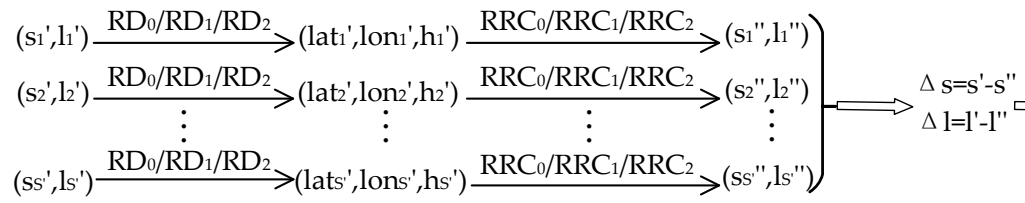

Output :

maximum error, root mean square error of range, azimuth and plane, respectively

$$
\begin{aligned}
& \text { Step 5: Positioning accuracy verification of RPC model } \\
& \text { Number of artificial real measurement GCPs: N } \\
& \Delta X=\sqrt{\sum_{i=1}^{N}\left(S_{R P C i}-S_{i}\right) / N} \times R_{r} \quad \Delta Y=\sqrt{\sum_{i=1}^{N}\left(L_{R P C i}-L_{i}\right) / N} \times R_{a}
\end{aligned}
$$

where $\Delta X$ and $\Delta Y$ are the positioning accuracies in the range and azimuth directions, respectively; $S_{i}$ and $L_{i}$ are the image coordinates of a GCP in the SAR image; $S_{R P C i}$ and $L_{R P C i}$ are the image coordinates of a GCP calculated using the RPC model; $R_{r}$ and $R_{a}$ are the pixel spacings in the range and azimuth directions (meter/pixel), respectively, which can be obtained from the auxiliary file of GF-3 satellite image.

Figure 1. Step-by-step routine for the rational polynomial coefficient (RPC) model fitting and accuracy verification algorithm. Note: $\mathrm{SAR}=$ synthetic aperture radar; $\mathrm{DEM}=$ digital elevation model; $\mathrm{RD}=$ range Doppler; NCEP $=$ National Center for Environmental Prediction; CODE $=$ European Center for Orbit Determination; GCP = ground control point. 
The steps of the algorithm can be described as follows.

(1) Firstly, the input data sets, including the SAR image auxiliary file and DEM of the study area, were prepared;

(2) Then, a virtual plane grid was built according to the image size, the elevation range of the coverage area was interpolated based on the DEM data and RD model, and layering processing was performed in the elevation direction to obtain a virtual space control grid. The number of layers was required to be greater than three to prevent the design matrix from becoming ill-conditioned [1]. Next, a virtual space check grid was constructed by interpolating between the centers of four adjacent virtual control points and the two adjacent elevation layers;

(3) The atmospheric propagation delay values were corrected at all virtual grid points using two plans: one in which the atmospheric delay correction value at the point at the center of the scene and the average elevation of the coverage area were employed to correct the atmospheric propagation delay at all virtual points, and one in which the atmospheric delay correction value at each virtual grid point was used to correct its own atmospheric delay;

(4) The RPC model was fitted separately using the original RD model, RD model modified using atmospheric delay correction plan 1 , and RD model modified using atmospheric delay correction plan 2, and then the fitting accuracy was evaluated. A detailed flowchart of the RPC model fitting accuracy evaluation is shown in Figure 2.

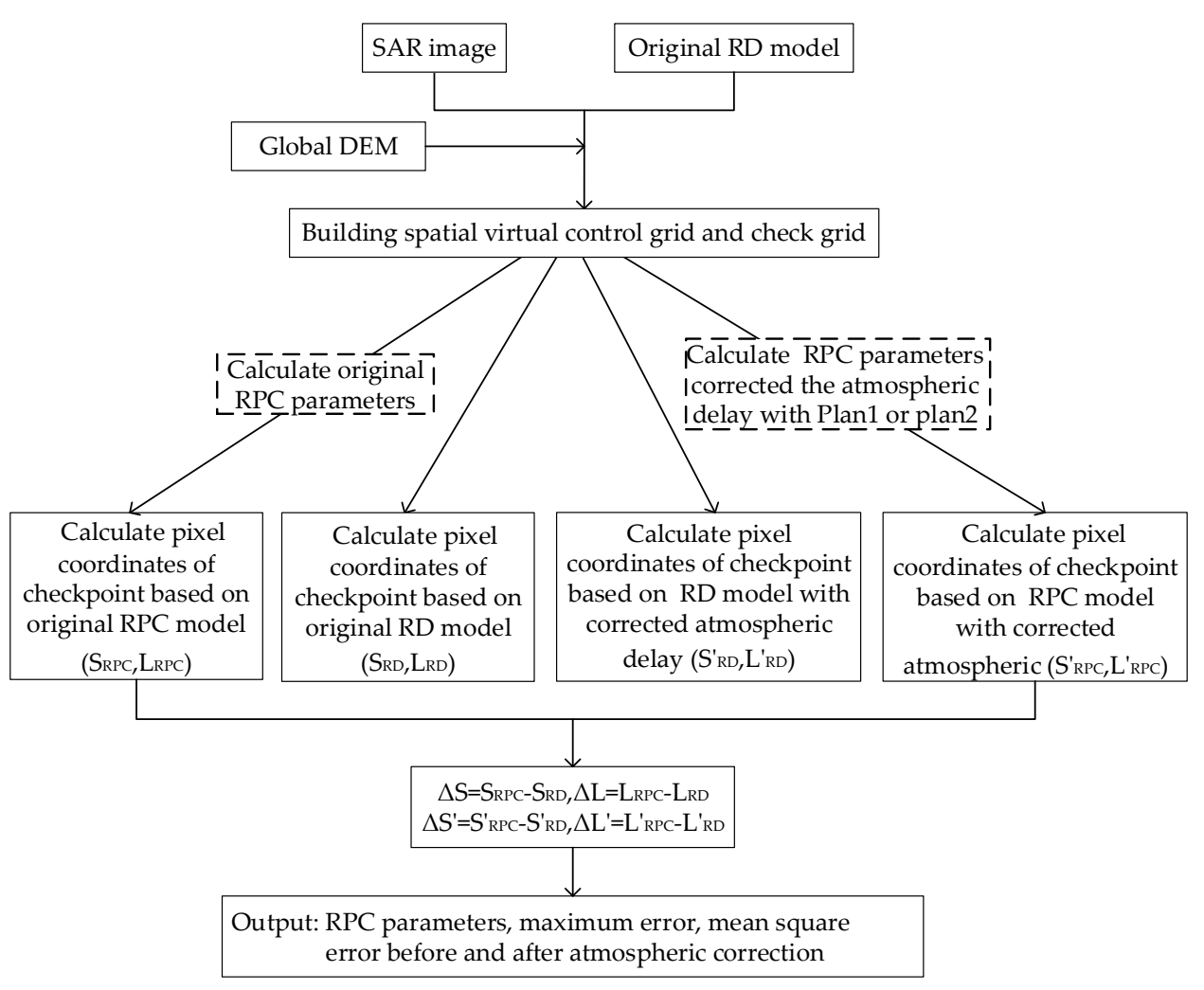

Figure 2. Flowchart of RPC fitting accuracy evaluation.

(5) The positioning accuracies of the abovementioned RPC models were evaluated based on the measured GCPs of the SAR image coverage area. To ensure the reliability of the verification accuracy, the root mean square error (RMSE) of multiple control points in one image was calculated using the equation in step $5, \Delta X$ and $\Delta Y$ as an evaluation index of the geometric positioning accuracy of the RPC model. 


\section{Results and Discussion}

\subsection{Experimental Data}

In this study, GF-3 images of four imaging modes, namely Spot-Light (1 m resolution), Ultra-Fine-Strip ( $3 \mathrm{~m}$ resolution), Fine-Strip-I (5 m resolution), and Fine-Strip-II (10 m resolution), were used as experimental data. To verify the positioning accuracy of the RPC model considering the atmospheric propagation delay, GF-3 satellite images and GCP data from Anping, Zhanjiang, Xianning, Zhangye, and Inner Mongolia in China were used. The GCPs were derived from global navigation satellite system (GNSS) receivers, which were selected in locations with obvious features, such as the intersections of pathways. Centimeter-level positioning accuracy could be achieved using real-time kinematic static observation technology. The details of the GF-3 satellite images are provided in Table 1, and the distribution of the experimental data and GCPs is depicted in Figure 3.

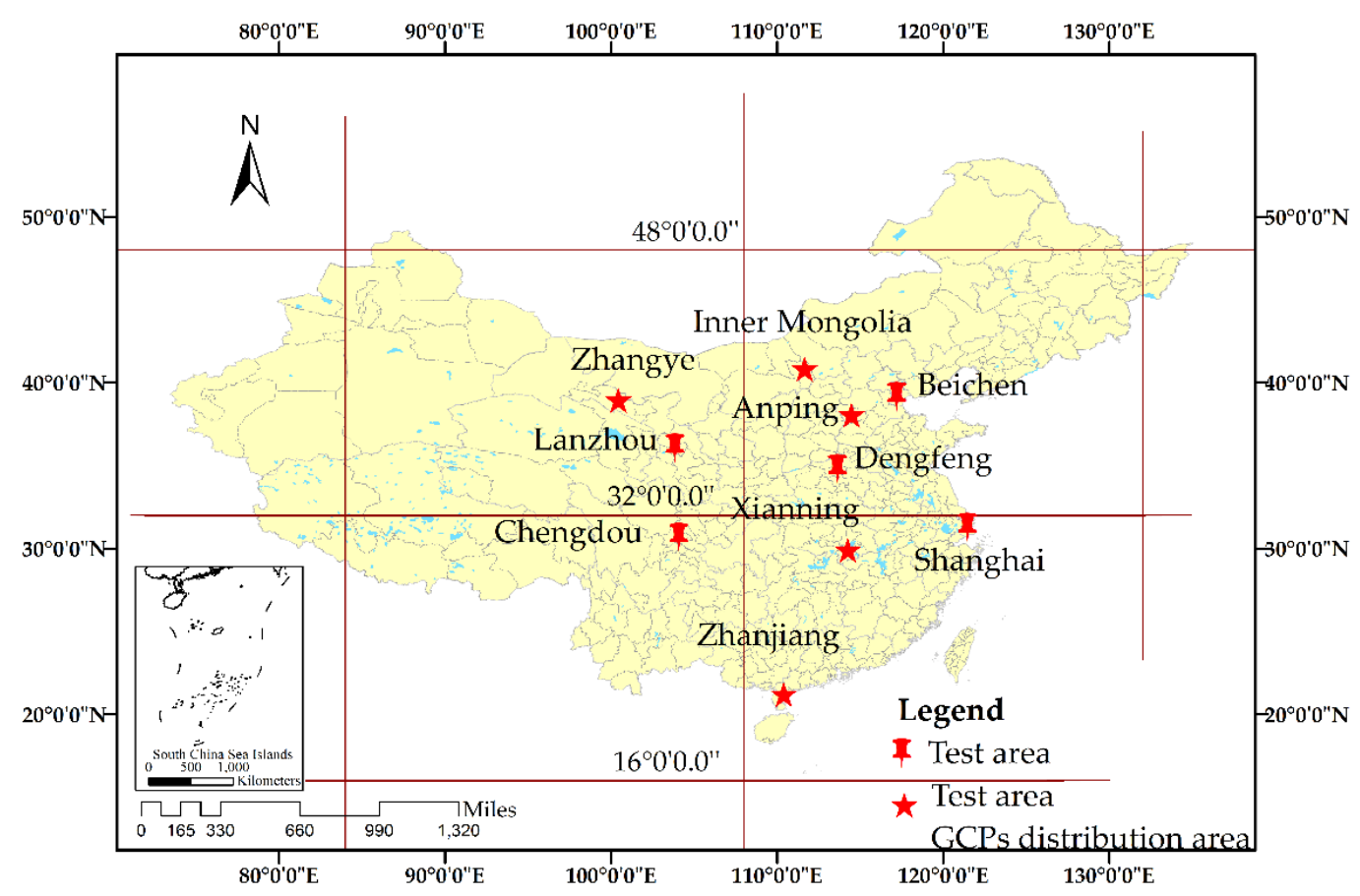

Figure 3. Distribution of the experimental data and GCPs.

Table 1. GF-3 data information of the study area.

\begin{tabular}{ccccccc}
\hline Imaging Mode & ID of Image & Imaging Region & Imaging Date & $\mathbf{R}_{\boldsymbol{r}}$ & $\mathbf{R}_{\boldsymbol{a}}$ & Number of GCPs \\
\hline \multirow{2}{*}{ SL } & CD-3076 & Chengdou & 8 January 2017 & 0.5621 & 0.3126 & \\
& DF-6954 & Dengfeng & 7 August 2018 & 0.5621 & 0.3125 & \\
& LZ-4905 & Lanzhou & 6 August 2018 & 0.5621 & 0.3443 & \\
\hline \multirow{2}{*}{ UFS } & BC-1094 & Beichen & 18 September 2016 & 1.1242 & 1.7308 & \\
& ZJ-8807 & Zhanjiang & 12 August 2017 & 1.1242 & 1.7327 & \\
& SH-4753 & Shanghai & 27 January 2017 & 1.1242 & 1.7292 & \\
\hline \multirow{5}{*}{ FSI } & AP-6616 & Anping & 19 February 2017 & 2.2484 & 2.8110 & 12 \\
& AP-4130 & Anping & 5 May 2017 & 1.1242 & 2.6183 & 6 \\
& ZJ-5851 & Zhanjiang & 25 January 2017 & 2.2484 & 2.8109 & \\
& XN-3579 & Xianning & 11 January 2017 & 2.2484 & 2.8139 & 6 \\
& NM-3601 & Neimeng & 11 January 2017 & 2.2484 & 2.8098 & 13 \\
& NM-2661 & Neimeng & 28 July 2017 & 1.1242 & 2.5915 & 10 \\
& ZY-7668 & Zhangye & 18 May 2019 & 2.2484 & 2.8252 & \\
\hline \multirow{2}{*}{ FSII } & CD-7465 & Chengdou & 3 October 2017 & 2.2484 & 4.7566 & \\
& ZJ-5492 & Zhanjiang & 6 October 2017 & 2.2484 & 4.7710 & 8 \\
& ZY-0316 & Zhangye & 22 January 2019 & 2.2484 & 4.7576 & 7 \\
\hline
\end{tabular}




\subsection{Experimental Results and Analysis}

To evaluate the feasibility of replacing the range Doppler equation of spaceborne SAR by considering the atmospheric delay with the RPC model, the following three experiments were conducted. (1) For the terrain-independent calculation algorithm of the RPC model of spaceborne SAR, the effects of the virtual grid pattern, number of elevation layers, and orbit fitting order on the fitting accuracy of the RPC model were analyzed. (2) Based on the appropriate grid pattern, number of elevation layers, and orbital fitting order, the fitting accuracy of the RPC model was assessed using the two proposed atmospheric delay correction plans. (3) Based on the fitting results of the RPC models, with and without considering the atmospheric delay of the radar signal, combined with the GCP data in the image coverage area, the positioning accuracy of the RPC model considering the atmospheric delay was verified.

\subsubsection{Impact of RPC Model Fitting Parameter}

\section{(1) Grid Size}

Assuming there to be five elevation layers, six different grid styles were set (style 1: 4000 pixels $\times$ 4000 pixels; style 2: 2000 pixels $\times 2000$ pixels; style 3: 1000 pixels $\times 1000$ pixels; style 4: 500 pixels $\times$ 500 pixels; style 5: 200 pixels $\times 200$ pixels; style 6: 100 pixels $\times 100$ pixels), here "N pixels $\times$ N pixels" refers to a grid spacing of $\mathrm{N}$ pixels, or a grid size. We take $\mathrm{N}$ pixels as the sampling interval in the range direction and azimuth direction, and take the sampling points as the virtual control points. Based on the fine-strip product data of the GF-3 satellite, DEM data with $30 \mathrm{~m}$ resolution, and the RPC fitting method described in Section 3, the influence of the grid size on the fitting accuracy of the RPC model was analyzed. The experimental results are shown in Figure 4a.

\section{(2) Number of Elevation Layers}

Using the appropriate grid pattern identified in the previous step, the number of different elevation layers (two, three, four, five, or six) was set to evaluate the influence of the number of elevation layers on the fitting accuracy of the RPC model. The experimental results are presented in Figure $4 \mathrm{~b}$.

\section{(3) Orbit Fitting Order}

With the appropriate grid pattern and number of elevation layers, different orders of satellite orbital fitting (third, fourth, or fifth order) were set to analyze the influence of the orbit fitting order on the fitting accuracy of the RPC model. The experimental results are depicted in Figure 4c.

Figure 4 shows that as the control grid becomes smaller and the number of elevation layers increases, the plane RMSE of the checkpoint gradually decreases. Eventually, the accuracy of the RPC model becomes close to that of the rigorous imaging geometry model. As the orbital parameter fitting order increases, the plane RMSE of the checkpoint gradually increases. To balance the fitting accuracy and computational efficiency of the RPC model, we set the grid size to 500 pixels $\times 500$ pixels, the number of elevation layers to five, and the orbit fitting order to third-order for the subsequent study.

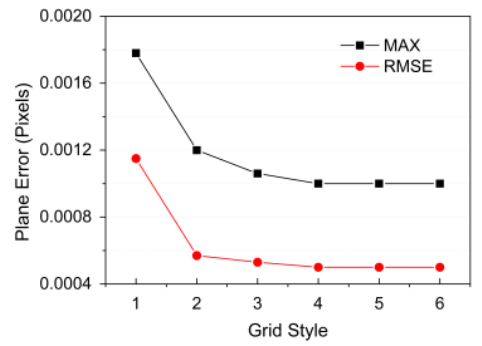

(a)

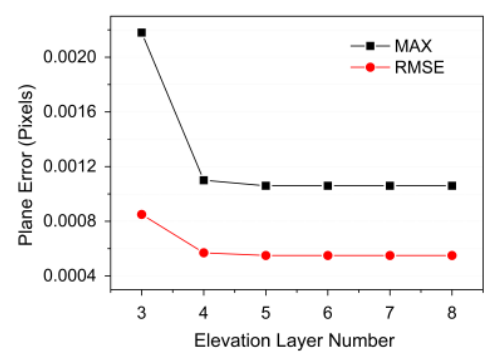

(b)

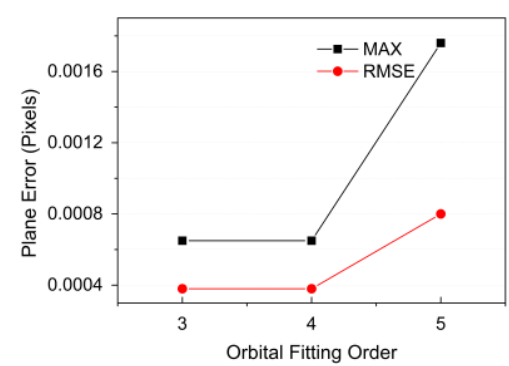

(c)

Figure 4. Impacts of RPC model fitting parameters: (a) grid size; (b) number of elevation layers; (c) orbit fitting order. 


\subsubsection{RPC Model Fitting Accuracy Evaluation}

Based on the atmospheric propagation delay correction model described in this paper combined with the meteorological data stored by the NCEP every $6 \mathrm{~h}$, the global ionospheric map provided by CODE every $1 \mathrm{~h}$, and the average elevation and size of the image coverage area provided by the GF-3 image auxiliary file, the atmospheric propagation delay correction of the radar signal was calculated to analyze the influence of atmospheric propagation delay correction on the RPC fitting accuracy.

In this study, atmospheric propagation delay correction of the radar signal was performed using two methods: one with a GF-3 image of a single scene and unique atmospheric delay correction parameters (plan 1) and one with an image of a single scene and spatially varying atmospheric delay correction parameters (plan 2). The two plans can be described as follows. In plan 1, the atmospheric delay correction at the point at the center of the scene and the average elevation of the coverage area are calculated to correct the atmospheric propagation delay at all virtual grid points. In plan 2, the atmospheric propagation delay correction value is calculated at each virtual grid point to correct its own atmospheric delay. The delay results are presented in Table 2.

Table 2. Atmospheric delay correction value calculation results.

\begin{tabular}{|c|c|c|c|c|c|c|c|c|}
\hline \multirow{2}{*}{ Topography } & \multirow{2}{*}{ Image ID } & \multicolumn{2}{|c|}{ Incidence Angle $\left({ }^{\circ}\right)$} & \multicolumn{3}{|c|}{ DEM (m) } & \multicolumn{2}{|c|}{$\Delta \mathrm{R}_{\text {delay }}(\mathrm{m})$} \\
\hline & & Near-Range & Far-Range & Minimum & Maximum & Mean & Plan 1 & Plan 2 \\
\hline \multirow{8}{*}{$\begin{array}{l}\text { Plains and } \\
\text { mountains }\end{array}$} & SH-4753 & 45.699 & 47.142 & -30 & 36 & 0.00 & -3.611 & $-3.678--3.544$ \\
\hline & AP-4130 & 26.182 & 29.657 & -28 & 65 & 11.66 & -2.825 & $-2.825--2.712$ \\
\hline & AP-6616 & 44.740 & 47.334 & -27 & 72 & 23.54 & -3.504 & $-3.623--3.399$ \\
\hline & BC-1094 & 19.759 & 22.199 & -29 & 81 & 13.68 & -2.813 & $-2.852--2.768$ \\
\hline & ZJ-8807 & 32.375 & 34.393 & -30 & 169 & 24.05 & -3.185 & $-3.258--3.071$ \\
\hline & ZJ-5851 & 46.510 & 48.971 & -35 & 350 & 23.03 & -3.795 & $-3.919-3.587$ \\
\hline & XN-3579 & 44.777 & 47.362 & -21 & 641 & 111.59 & -3.393 & $-3.771-3.281$ \\
\hline & CD-3076 & 26.160 & 27.019 & 426 & 975 & 695.23 & -2.522 & $-2.613--2.400$ \\
\hline \multirow{8}{*}{ Plateau } & ZJ-5492 & 31.291 & 38.155 & -33 & 1271 & 22.52 & -3.531 & $-3.702-2.824$ \\
\hline & DF-6954 & 28.717 & 29.698 & 312 & 1470 & 446.73 & -2.865 & $-2.943--2.477$ \\
\hline & NM-3601 & 44.746 & 47.334 & 976 & 1970 & 1052.78 & -3.028 & $-3.516--2.665$ \\
\hline & NM-2661 & 23.865 & 27.674 & 952 & 2214 & 990.42 & -2.561 & $-2.617-2.136$ \\
\hline & ZY-7668 & 34.665 & 37.920 & 1371 & 3222 & 1498.87 & -2.536 & $-2.636-2.041$ \\
\hline & ZY-0316 & 30.894 & 37.837 & 1300 & 4769 & 2092.58 & -2.28 & $-2.569--1.568$ \\
\hline & LZ-4905 & 41.035 & 41.745 & 1478 & 2150 & 1606.19 & -3.012 & $-3.095--2.780$ \\
\hline & CD-7465 & 31.294 & 37.718 & 390 & 4931 & 810.42 & -3.111 & $-3.212-1.521$ \\
\hline
\end{tabular}

In Table 2, the calculation results for plan 1 show that the maximum slant range correction value at the center point among the different images is $-3.795 \mathrm{~m}$, and the delay error in the ground range direction was calculated based on the incident angle of the image as approximately $-5 \mathrm{~m}$, which indicates that the atmospheric delay error has a significant influence on the positioning accuracy. The calculation results for plan 2 show that the atmospheric delay correction values of the virtual grid points with different spatial distributions on a scene image are different, and the slant range correction values of the virtual grid points in all of the image scenes are between $1.521 \mathrm{~m}$ and $-3.919 \mathrm{~m}$. Thus, the atmospheric delay correction values differ between imaging angles and imaging regions. It was, therefore, concluded that the atmospheric propagation delay is mainly affected by the radar incidence angle and the topography of the study area.

To analyze the main influencing factors of the atmospheric propagation delay in the SAR image scenes further, we visualized the atmospheric propagation delay correction values of the virtual grid points calculated using plan 2, assuming this to be 0 pixels in the azimuth direction and sampling every 500 pixels in the range direction, with five elevation layers. The trend of the atmospheric delay correction value in the range direction is shown in Figure 5. To analyze the influence of the elevation on the atmospheric propagation delay, we increased the number of elevation layers of the virtual control grid to 300 separately in the plains and mountains area (ZJ-5851) and the plateau area (ZY-7668). The maximum variation $\Delta \varphi$ of the corresponding incident angle at the same sampling point in each elevation layer in the experimental region is shown in Figure 6, where the orbital height of the satellite 
is known to be approximately $755 \mathrm{~km}$. Here, $\Delta \varphi$ was approximated using Equation (9), and was found to be less than $0.03^{\circ}$ in the ZJ-5851 image coverage area and less than $0.15^{\circ}$ in the ZY-7668 image coverage area. Thus, the variation of the incident angle in the elevation direction in the study area is negligible, and the same sampling point can be selected in each elevation layer to analyze the atmospheric propagation delay law as a function of elevation, as shown in Figure 7.

$$
\Delta \varphi<\frac{H_{\max }}{H_{S}} \times \frac{180^{\circ}}{\pi}
$$

where $H_{S}$ is the orbital height of the GF-3 satellite; $H_{\max }$ and $H_{\min }$ are the maximum and minimum elevations of the image coverage area, respectively.

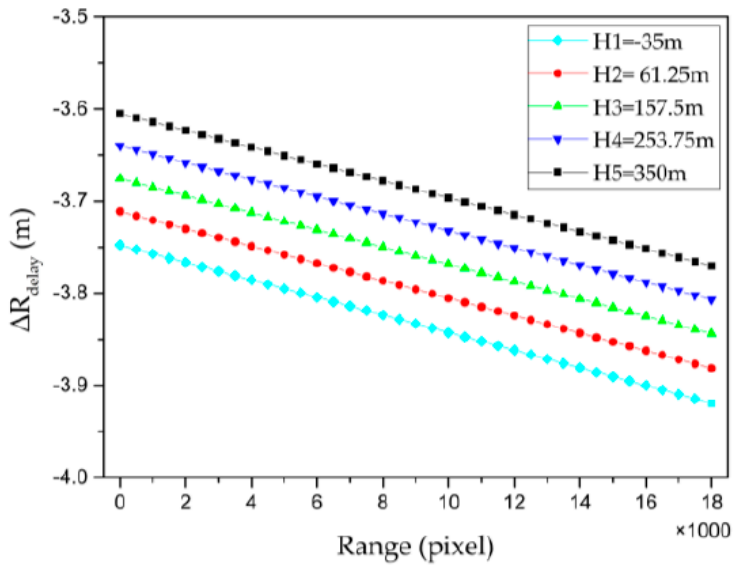

(a)

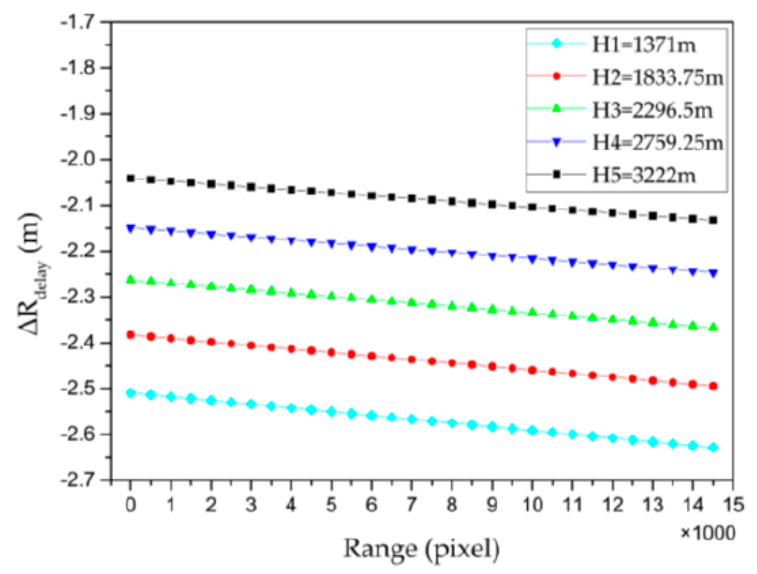

(b)

Figure 5. Trend of atmospheric delay correction value in the range direction: (a) plains and mountains area (ZJ-5851); (b) plateau area (ZY-7668).

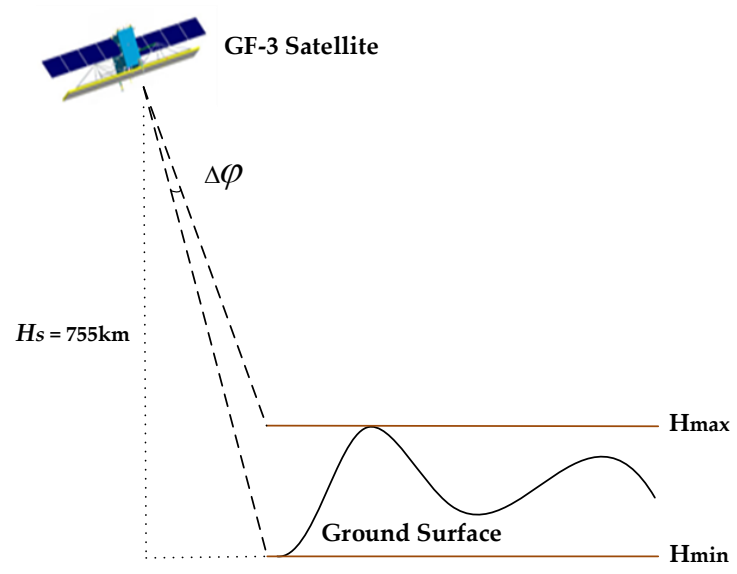

Figure 6. Variation of incident angle in the elevation direction in the study area.

Figure 5 shows that the atmospheric delay and incident angle are approximately linearly related at the same elevation level. The absolute value of the atmospheric propagation delay correction gradually increases as the range increases.

Some researchers have demonstrated that the elevation-atmospheric delay relationship is usually a linear or exponential function [20,21]. Figure 7a shows that in the plains and mountains area, the elevation-atmospheric delay relationship is approximately linear. Meanwhile, Figure $7 \mathrm{~b}$ reveals that in the plateau region, the elevation-atmospheric delay relationship is also approximately linear. 


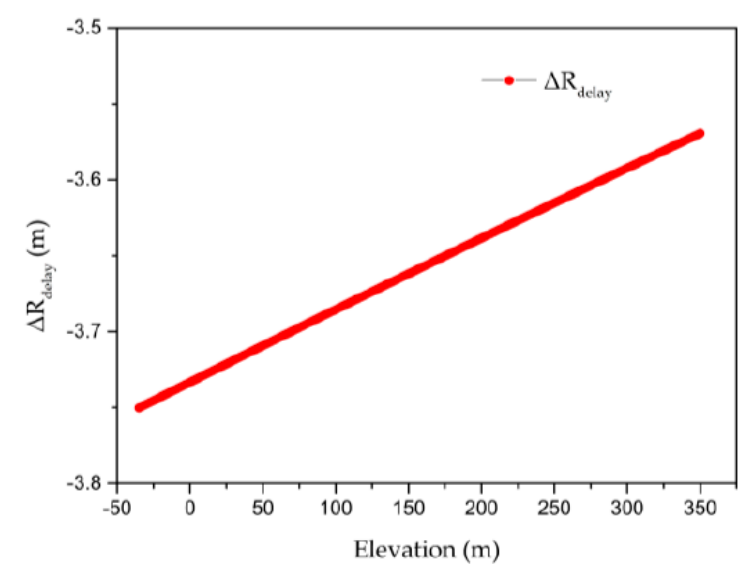

(a)

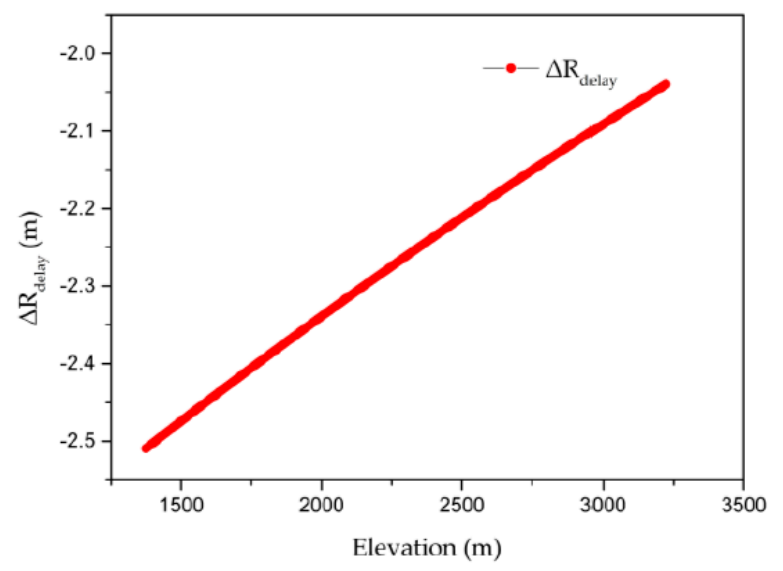

(b)

Figure 7. Atmospheric delay correction as a function of elevation: (a) plains and mountains area (ZJ-5851); (b) plateau area (ZY-7668).

According to the RPC model fitting parameter impact analysis results in Section 4.2.1, we established a control grid by setting the grid size to 500 pixels $\times 500$ pixels and the number of elevation layers to five. The checkpoint was determined based on the centers of the four adjacent control points and two adjacent elevation layers, and the fitting accuracy of the RPC model was evaluated after correcting the atmospheric delay grid by grid. The results are presented in Table 3.

In general, as the plane error of the checkpoint does not exceed $5 \%$ of the pixels, we believe that the RPC model can be used instead of the RD model for photogrammetric processing of spaceborne SAR satellites. Table 3 shows that in the same scene, both the uncorrected atmospheric delay and atmospheric delay correction with plan 1 yield exactly the same RPC model fitting accuracy, and that the maximum plane RMSE of the checkpoint is 0.00356 pixels. For the atmospheric delay correction with plan 2, the fitting accuracy of the RPC model is very approximate, so both approaches achieve precision better than $1 \%$ of a pixel. This indicates that the RPC model still provides high accuracy for the RD model after correcting the atmospheric delay using two plans, in plain, mountainous, and plateau areas.

The reason for obtaining these experimental results can be confirmed by research that has shown that the third-order RPC model can better fit a function whose highest power is no more than 5 [22]. For a single-scene SAR image, plan 1 uses a unique atmospheric delay correction parameter to correct the atmospheric propagation delay at all virtual grid points, which is equivalent to adding the atmospheric propagation delay as a constant function to the slant range $(\mathrm{R})$ term of the RD model. Therefore, the fitting accuracy of the RPC model is unchanged. Meanwhile, plan 2 corrects the atmospheric propagation delay at all virtual grid points with the correction parameters of the corresponding virtual grid points, which is equivalent to adding the atmospheric delay correction model to the $\mathrm{R}$ term with the elevation as the dependent variable. Figure 7a shows that the elevation-atmospheric delay function is approximately linear in the plains and mountains area, so it can be correctly fitted with a third-order RPC model. Meanwhile, Figure $7 \mathrm{~b}$ shows that in the plateau area, the elevation-atmospheric delay function also shows an approximate linear relationship, which indicates that RPC model can not only fit complex terrain, but also fit the terrain-related atmospheric propagation delay. 
Table 3. Fitting accuracy of the RPC model. Note: Max. = maximum; RMSE = root mean square error.

\begin{tabular}{|c|c|c|c|c|c|c|c|c|c|c|c|c|c|c|}
\hline \multirow{3}{*}{ Topography } & \multirow{3}{*}{ Image ID } & \multirow{3}{*}{ Compensation Method } & \multicolumn{6}{|c|}{ Control Point Error (Pixels) } & \multicolumn{6}{|c|}{ Check Point Error (Pixels) } \\
\hline & & & \multicolumn{2}{|c|}{ Sample } & \multicolumn{2}{|c|}{ Line } & \multicolumn{2}{|c|}{ 2-D } & \multicolumn{2}{|c|}{ Sample } & \multicolumn{2}{|c|}{ Line } & \multicolumn{2}{|c|}{ 2-D } \\
\hline & & & Max. & RMSE & Max. & RMSE & Max. & RMSE & Max. & RMSE & Max. & RMSE & Max. & RMSE \\
\hline \multirow{16}{*}{ Plains and Mountains } & \multirow{2}{*}{ SH-4753 } & Uncorrected/Plan 1 & 0.00041 & 0.00013 & 0.00013 & 0.00007 & 0.00041 & 0.00015 & 0.00029 & 0.00012 & 0.00015 & 0.00007 & 0.0003 & 0.00014 \\
\hline & & Plan 2 & 0.00041 & 0.00013 & 0.00013 & 0.00007 & 0.00041 & 0.00015 & 0.00029 & 0.00012 & 0.00015 & 0.00007 & 0.0003 & 0.00014 \\
\hline & \multirow{2}{*}{ AP-4130 } & Uncorrected/Plan 1 & 0.0122 & 0.00364 & 0.0032 & 0.0012 & 0.01245 & 0.00383 & 0.00933 & 0.00338 & 0.00219 & 0.00111 & 0.0095 & 0.00356 \\
\hline & & Plan 2 & 0.01243 & 0.00366 & 0.0032 & 0.0012 & 0.01268 & 0.00385 & 0.00944 & 0.0034 & 0.00219 & 0.00111 & 0.00961 & 0.00357 \\
\hline & \multirow{2}{*}{ AP-6616 } & Uncorrected/Plan 1 & 0.00172 & 0.00059 & 0.00009 & 0.00004 & 0.00172 & 0.00059 & 0.00126 & 0.00055 & 0.00012 & 0.00005 & 0.00126 & 0.00055 \\
\hline & & Plan 2 & 0.00171 & 0.00059 & 0.00009 & 0.00004 & 0.00171 & 0.0006 & 0.00127 & 0.00055 & 0.00012 & 0.00005 & 0.00127 & 0.00055 \\
\hline & \multirow[b]{2}{*}{ BC-1094 } & Uncorrected/Plan 1 & 0.00074 & 0.00025 & 0.00012 & 0.00007 & 0.00075 & 0.00026 & 0.00053 & 0.00022 & 0.00016 & 0.00007 & 0.00055 & 0.00024 \\
\hline & & Plan 2 & 0.00073 & 0.00026 & 0.00012 & 0.00007 & 0.00074 & 0.00027 & 0.00053 & 0.00023 & 0.00016 & 0.00007 & 0.00055 & 0.00025 \\
\hline & \multirow{2}{*}{ ZJ-8807 } & Uncorrected/Plan 1 & 0.00077 & 0.00024 & 0.0012 & 0.00047 & 0.00132 & 0.00052 & 0.00059 & 0.00022 & 0.00077 & 0.00043 & 0.00084 & 0.00048 \\
\hline & & Plan 2 & 0.00078 & 0.00024 & 0.0012 & 0.00047 & 0.00132 & 0.00052 & 0.00059 & 0.00022 & 0.00077 & 0.00043 & 0.00084 & 0.00048 \\
\hline & \multirow{2}{*}{ ZJ-5851 } & Uncorrected/Plan 1 & 0.00276 & 0.0009 & 0.00164 & 0.00063 & 0.00318 & 0.0011 & 0.00203 & 0.00083 & 0.001 & 0.00058 & 0.00218 & 0.00102 \\
\hline & & Plan 2 & 0.00276 & 0.0009 & 0.00164 & 0.00063 & 0.00316 & 0.0011 & 0.00203 & 0.00083 & 0.001 & 0.00058 & 0.00218 & 0.00102 \\
\hline & \multirow{2}{*}{ XN-3579 } & Uncorrected/Plan 1 & 0.00084 & 0.0002 & 0.00031 & 0.00007 & 0.00089 & 0.00021 & 0.00056 & 0.00018 & 0.0002 & 0.00007 & 0.00059 & 0.0002 \\
\hline & & Plan 2 & 0.00083 & 0.00019 & 0.00031 & 0.00007 & 0.00088 & 0.00021 & 0.00054 & 0.00018 & 0.0002 & 0.00007 & 0.00057 & 0.00019 \\
\hline & \multirow{2}{*}{ CD-3076 } & Uncorrected/Plan 1 & 0.00015 & 0.00005 & 0.00066 & 0.00032 & 0.00066 & 0.00033 & 0.00012 & 0.00005 & 0.00093 & 0.00046 & 0.00093 & 0.00046 \\
\hline & & Plan 2 & 0.00015 & 0.00005 & 0.00066 & 0.00032 & 0.00066 & 0.00033 & 0.00012 & 0.00005 & 0.00093 & 0.00046 & 0.00093 & 0.00046 \\
\hline \multirow{16}{*}{ Plateau } & \multirow{2}{*}{ ZJ-5492 } & Uncorrected/Plan 1 & 0.00839 & 0.00207 & 0.00058 & 0.00014 & 0.00839 & 0.00207 & 0.00658 & 0.00192 & 0.00044 & 0.00013 & 0.00658 & 0.00193 \\
\hline & & Plan 2 & 0.00843 & 0.00209 & 0.00058 & 0.00014 & 0.00843 & 0.00209 & 0.0066 & 0.00194 & 0.00044 & 0.00013 & 0.0066 & 0.00194 \\
\hline & \multirow{2}{*}{ DF-6954 } & Uncorrected/Plan 1 & 0.00134 & 0.00046 & 0.00079 & 0.00039 & 0.00146 & 0.00061 & 0.00112 & 0.00044 & 0.00119 & 0.00043 & 0.00158 & 0.00061 \\
\hline & & Plan 2 & 0.00134 & 0.00047 & 0.00079 & 0.00039 & 0.00147 & 0.00061 & 0.00112 & 0.00044 & 0.00119 & 0.00043 & 0.00158 & 0.00062 \\
\hline & \multirow{2}{*}{ NM-3601 } & Uncorrected/Plan 1 & 0.00072 & 0.00018 & 0.00026 & 0.00011 & 0.00074 & 0.00021 & 0.00054 & 0.00017 & 0.00024 & 0.0001 & 0.00055 & 0.0002 \\
\hline & & Plan 2 & 0.00075 & 0.00019 & 0.00026 & 0.00011 & 0.00077 & 0.00022 & 0.00057 & 0.00017 & 0.00024 & 0.0001 & 0.00059 & 0.0002 \\
\hline & NMM 2661 & Uncorrected/Plan 1 & 0.00832 & 0.00179 & 0.00106 & 0.00041 & 0.00839 & 0.00184 & 0.00555 & 0.00165 & 0.00078 & 0.00038 & 0.00561 & 0.00169 \\
\hline & NM-2661 & Plan 2 & 0.00833 & 0.00179 & 0.00106 & 0.00041 & 0.00839 & 0.00184 & 0.00553 & 0.00165 & 0.00078 & 0.00038 & 0.00559 & 0.00169 \\
\hline & & Uncorrected/Plan 1 & 0.00111 & 0.00025 & 0.00167 & 0.00067 & 0.00185 & 0.00072 & 0.00073 & 0.00023 & 0.00113 & 0.00065 & 0.00134 & 0.00069 \\
\hline & ZY-7668 & Plan 2 & 0.00123 & 0.00026 & 0.00167 & 0.00071 & 0.00203 & 0.00075 & 0.00074 & 0.00023 & 0.00113 & 0.00065 & 0.00134 & 0.00069 \\
\hline & & Uncorrected/Plan 1 & 0.0106 & 0.00216 & 0.00141 & 0.00033 & 0.01061 & 0.00219 & 0.00756 & 0.00201 & 0.00094 & 0.00031 & 0.00756 & 0.00203 \\
\hline & ZY-0316 & Plan 2 & 0.0106 & 0.00215 & 0.00142 & 0.00033 & 0.01061 & 0.00217 & 0.00752 & 0.00199 & 0.00094 & 0.00031 & 0.00752 & 0.00202 \\
\hline & & Uncorrected/Plan 1 & 0.0018 & 0.00067 & 0.00133 & 0.00051 & 0.00224 & 0.00085 & 0.00149 & 0.00064 & 0.00158 & 0.00053 & 0.00216 & 0.00083 \\
\hline & LZ-4905 & Plan 2 & 0.00181 & 0.00067 & 0.00133 & 0.00051 & 0.00222 & 0.00085 & 0.0015 & 0.00064 & 0.00158 & 0.00053 & 0.00216 & 0.00083 \\
\hline & & Uncorrected/Plan 1 & 0.01 & 0.002 & 0.0067 & 0.00241 & 0.01113 & 0.00371 & 0.00826 & 0.00261 & 0.00469 & 0.00223 & 0.00897 & 0.00344 \\
\hline & CD-7465 & Plan 2 & 0.01 & 0.002 & 0.0067 & 0.00241 & 0.01112 & 0.0037 & 0.00827 & 0.00261 & 0.00469 & 0.00223 & 0.00899 & 0.00344 \\
\hline
\end{tabular}




\subsubsection{RPC Model Positioning Accuracy Verification}

From the perspective of RPC model application, we carried out RPC model positioning experiments to verify the effectiveness of the RPC model considering atmospheric propagation delay. Based on the experimental data of the GF-3 satellite and GCPs, geometric positioning verification experiments were performed for the four RPC models, and the results are shown in Table 4.

(1) Firstly, for the RPC model fitted using the original RD model without atmospheric delay correction, positioning accuracy verification was conducted;

(2) After correcting the systematic error in the slant range direction of the GF-3 SAR satellite, geometric positioning accuracy verification of the RPC model was performed. This systematic error was obtained by geometric calibration of the spaceborne SAR [23];

(3) After correcting the systematic error in the slant range direction of the GF-3 SAR satellite and correcting the atmospheric propagation delay error using plan 1, geometric positioning accuracy verification of the RPC model was performed;

(4) After correcting the systematic error in the slant range direction of the GF-3 SAR satellite and correcting the atmospheric propagation delay error using plan 2, geometric positioning accuracy verification of the RPC model was performed.

Table 4 shows that the positioning accuracy of the RPC model is between $21.241 \mathrm{~m}$ and 26.004 $\mathrm{m}$ without correction of system error and atmospheric delay error. After correcting the system error, the geometric positioning error of the RPC model was between $3.064 \mathrm{~m}$ and $5.529 \mathrm{~m}$. After correcting the atmospheric delay error using plans 1 and 2, respectively, the maximum geometric positioning error of the RPC model was found to be $2.948 \mathrm{~m}$ and $2.957 \mathrm{~m}$, and the positioning accuracy of a single scene image could be increased by $2.7 \mathrm{~m}$, such as for ZJ-5492. Thus, it is effective to improve the geometric positioning accuracy of the RPC model by correcting the atmospheric propagation delay in the process of fitting the RD model to the RPC model. Furthermore, the positioning accuracy of the four RPC models in the azimuth direction is almost unchanged, which indicates that the slant range error caused by the atmospheric propagation delay mainly affects the positioning accuracy in the range direction.

Figure 8 shows that the positioning error of all GCPs in the SAR image of a single scene is approximately $2.5 \mathrm{~m}$ after correcting the atmospheric propagation delay using plans 1 and 2 separately, and this remains relatively stable, which indicates that the geometric positioning results of the RPC model are reliable.

It is worth noting that although both plans are feasible, it is still difficult to produce a RPC model considering atmospheric delay directly and in real time in the SAR system, because sufficiently precise atmospheric models typically have latency. We may be able to choose a general model in which the atmospheric delay is a function of the SAR incidence angle and the elevation of a given point, in order to replace the precise atmospheric model [24]. This will be our next research direction.

Table 4. Geometric positioning accuracies of the four RPC models.

\begin{tabular}{cccccccccccccc}
\hline \multirow{2}{*}{ Image ID } & \multicolumn{10}{c}{ Correction Method } \\
\cline { 2 - 14 } & \multicolumn{3}{c}{ Uncorrected } & \multicolumn{1}{c}{ Correct System Error } & \multicolumn{3}{c}{ Plan 1 } & \multicolumn{3}{c}{ Plan 2 } \\
\cline { 2 - 14 } & Sample & Line & 2-D & Sample & Line & 2-D & Sample & Line & 2-D & Sample & Line & 2-D \\
\hline AP-6616 & 22.858 & 1.380 & 22.900 & 4.021 & 1.380 & 4.409 & 2.588 & 1.380 & 2.934 & 2.599 & 1.380 & 2.944 \\
AP-4130 & 21.619 & 1.000 & 21.642 & 2.782 & 1.000 & 3.064 & 1.878 & 1.000 & 2.129 & 1.871 & 1.000 & 2.123 \\
XN-3579 & 22.397 & 1.457 & 22.446 & 3.561 & 1.457 & 4.004 & 2.382 & 1.457 & 2.801 & 2.380 & 1.457 & 2.792 \\
NM-3601 & 22.460 & 0.118 & 22.496 & 3.623 & 0.117 & 3.941 & 2.110 & 0.117 & 2.459 & 0.510 & 0.118 & 2.486 \\
NM-2661 & 21.207 & 1.188 & 21.241 & 2.730 & 1.188 & 3.075 & 1.603 & 1.188 & 2.021 & 1.602 & 1.188 & 2.021 \\
ZY-7668 & 23.198 & 1.372 & 23.238 & 4.360 & 1.373 & 4.571 & 1.447 & 1.372 & 1.994 & 1.388 & 1.372 & 1.952 \\
ZJ-5492 & 25.899 & 2.333 & 26.004 & 5.013 & 2.333 & 5.529 & 1.600 & 2.332 & 2.828 & 1.885 & 2.333 & 2.999 \\
ZY-0316 & 24.983 & 1.001 & 25.003 & 4.096 & 1.000 & 4.217 & 1.184 & 1.000 & 1.550 & 1.166 & 1.000 & 1.536 \\
\hline
\end{tabular}




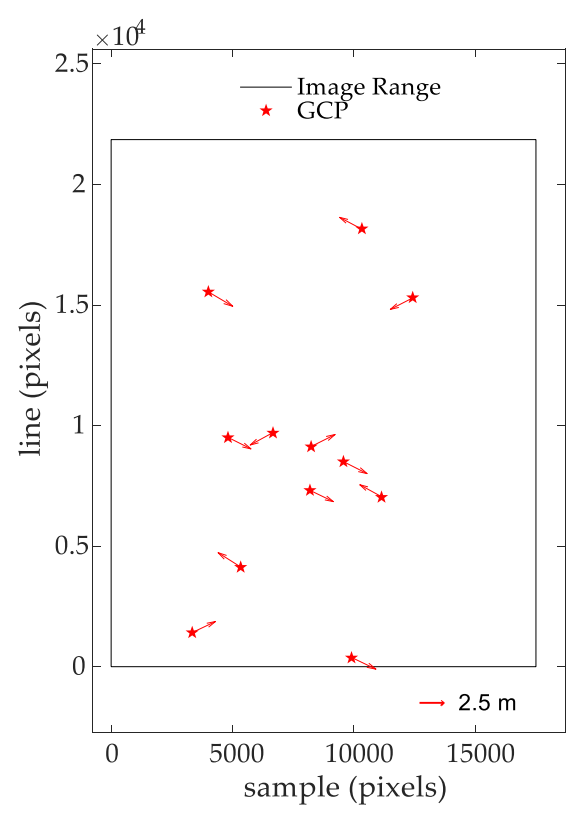

(a)

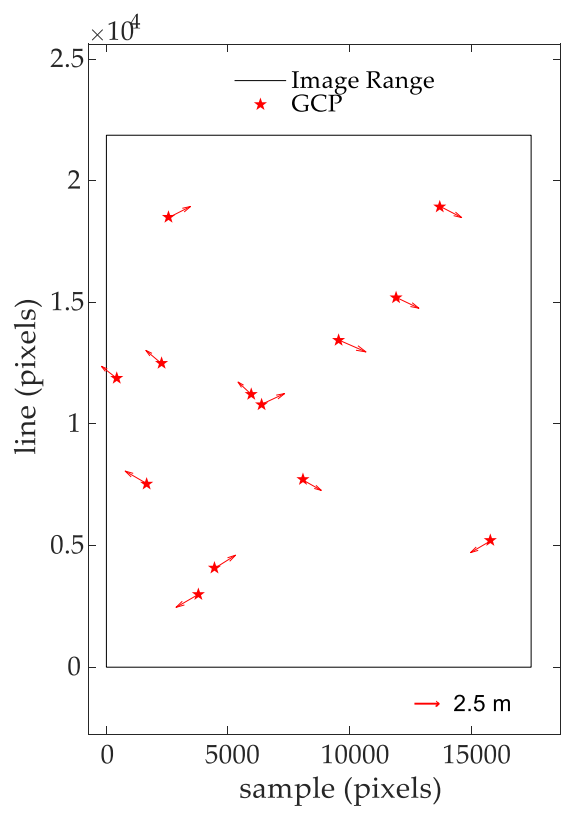

(c)

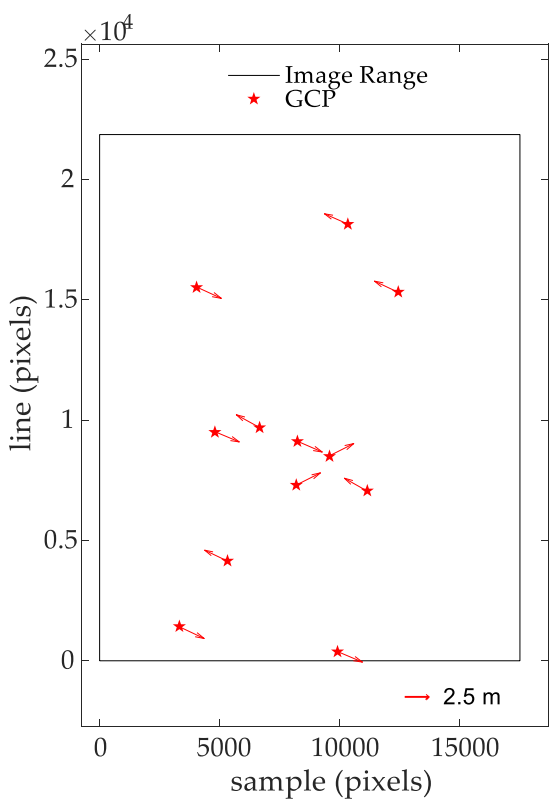

(b)

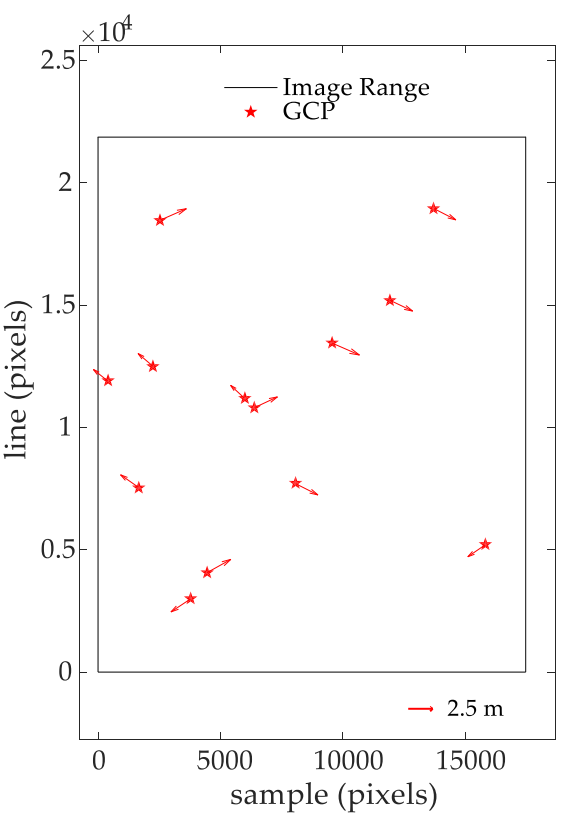

(d)

Figure 8. Positioning error distributions of all GCPs in one SAR image: (a) atmospheric delay corrected with plan 1 for image AP-6616; (b) atmospheric delay corrected with plan 2 for image AP-6616; (c) atmospheric delay corrected with plan 1 for image NM-3601; (d) atmospheric delay corrected with plan 2 for image NM-3601.

\section{Conclusions}

The atmospheric delays of SAR satellite radar signals can reach several meters, which is non-negligible in high-precision geometric positioning. Thus, two methods of atmospheric delay correction during RPC fitting of an RD model were designed and tested in this study. Based on the GF-3 SAR satellite data, a global digital elevation model, global meteorological data, and ground control data from several regions in China, the feasibility of the two methods was verified by conducting RPC model fitting and positioning experiments. We found that the RPC model exhibited high substitution 
and positioning accuracies after using plan 1 or plan 2 to correct the atmospheric propagation delay, in both the plains and mountains area where the relative height difference is less than $1000 \mathrm{~m}$, and in the plateau area where the relative height difference is more than $1000 \mathrm{~m}$. Therefore, it is feasible to use plan 1 or plan 2 to correct the atmospheric delay error under any terrain conditions. Moreover, the geometric positioning accuracy of the RPC model after correcting the atmospheric delay was improved to better than $3 \mathrm{~m}$.

Author Contributions: G.Z., Y.H., R.Z., S.H. and P.J. conceived and designed the experiments. G.Z., S.H., and R.Z. performed the experiments. S.H., R.Z. and P.Z. analyzed the data. S.H. wrote the paper. All authors have read and agreed to the published version of the manuscript.

Funding: This research was supported by the Key Research and Development Program of Ministry of Science and Technology, grant 2016YFB0500801; the National Natural Science Foundation of China, grants 91538106, 41501503, 41601490, and 41501383; and the Open Research Fund of State Key Laboratory of Information Engineering in Surveying, Mapping, and Remote Sensing, grant 15E02. The authors also thank the anonymous reviewers for their constructive comments and suggestions.

Conflicts of Interest: The authors declare no conflict of interest.

\section{References}

1. Tao, C.V.; Hu, Y. A comprehensive study of the rational function model for photogrammetric processing. Photogramm. Eng. Remote Sens. 2001, 67, 1347-1357.

2. Toutin, T. Review article: Geometric processing of remote sensing images: Models, algorithms and methods. Int. J. Remote Sens. 2004, 25, 1893-1924. [CrossRef]

3. Zhang, G.; Fei, W.B.; Li, Z.; Zhu, X.; Li, D. Evaluation of the RPC model for spaceborne SAR imagery. Photogramm. Eng. Remote Sens. 2010, 76, 727-733. [CrossRef]

4. Eftekhari, A.; Saadatseresht, M.; Motagh, M. A study on rational function model generation for Terrasar-X imagery. Sensors. 2013, 13, 12030-12043. [CrossRef] [PubMed]

5. Zhang, G.; Zhu, X.Y. A study of the RPC model of Terrasar-X and Cosmo-Skymed SAR imagery. Remote Sens. Spat. Inf. Sci. 2008, 37, 321-324.

6. Wang, T.Y.; Zhang, G.; Yu, L.; Zhao, R.; Deng, M.; Xu, K. Multi-mode gf-3 satellite image geometric accuracy verification using the RPC model. Sensors 2017, 17, 2005. [CrossRef] [PubMed]

7. Jiao, N.G.; Wang, F.; You, H.J.; Qiu, X.L.; Yang, M.D. Geo-positioning accuracy improvement of multi- mode gf-3 satellite SAR imagery based on error sources analysis. Sensors 2018, 18, 2333. [CrossRef]

8. Zhao, R.S.; Jiang, Y.H.; Zhang, G.; Deng, M.J.; Yang, F. Geometric accuracy evaluation of YG-18 satellite imagery based on RFM. Photogramm. Rec. 2017, 32, 33-47. [CrossRef]

9. Huang, Z.W.; He, S. A method for improving positioning accuracy of SAR imagery based on RFM. In Proceedings of the 4 th International Conference on Digital Manufacturing \& Automation, Qindao, China, 29-30 June 2013; pp. 43-46.

10. Liu, X.F.; Liu, J.Y.; Hong, W. The analysis of the precision in spaceborne SAR image location. J. Remote Sens. 2006, 10, 76-81.

11. Eineder, M.; Minet, C.; Steigenberger, P.; Cong, X.; Fritz, T. Imaging Geodesy-Toward Centimeter-Level Ranging Accuracy with TerraSAR-X. IEEE Trans. Geosci. Remote Sens. 2011, 49, 661-671. [CrossRef]

12. Cong, X.; Balss, U.; Eineder, M.; Fritz, T. Imaging geodesy-Centimeter-Level ranging accuracy with TerraSAR-X: An Update. IEEE Geosci. Remote Sens. Lett. 2012, 9, 948-952. [CrossRef]

13. Davis, J.L.; Herring, T.A.; Shapiro, I.I.; Rogers, A.E.E.; Elgered, G. Geodesy by radio interferometry: Effects of atmospheric modeling errors on estimates of baseline length. Radio Sci. 2016, 20, 1593-1607. [CrossRef]

14. Puyssegur, B.; Michel, R.; Avouac, J.P. Tropospheric phase delay in interferometric synthetic aperture radar estimated from meteorological model and multispectral imagery. J. Geophys. Res. Solid Earth. 2007, 112, 12. [CrossRef]

15. Smith, E.K.; Weintraub, S. The constants in the equation for atmospheric refractive index at radio frequencies. Proc. IRE 1953, 41, 1035-1037. [CrossRef]

16. Solheim, F.S.; Vivekanandan, J.; Ware, R.H.; Rocken, C. Propagation delays induced in GPS signals by dry air, water vapor, hydrometeors, and other particulates. J. Geophys. Res. 1999, 104, 9663-9670. [CrossRef] 
17. Skone, S.; Cannon, M.E. Ionospheric effects on differential GPS applications during auroral substorm activity. ISPRS J. Photogramm. Remote Sens. 1999, 54, 279-288. [CrossRef]

18. Curlander, J.C. Location of spaceborne SAR imagery. IEEE Trans. Geosci. Remote Sens. 1982, 3, 359-364. [CrossRef]

19. Grodecki, J.; Dial, G. Block adjustment of high-resolution satellite images described by rational polynomials. Photogramm. Eng. Remote Sens. 2003, 69, 59-68. [CrossRef]

20. Cavalie, O.; Doin, M.P.; Lasserre, C.; Briole, P. Ground motion measurement in the Lake Mead area, Nevada, by differential synthetic aperture radar interferometry time series analysis: Probing the lithosphere rheological structure. J. Geophys. Res. Solid Earth 2007, 112, B03403. [CrossRef]

21. Onn, F.; Zebker, H.A. Correction for interferometric synthetic aperture radar atmospheric phase artifacts using time series of zenith wet delay observations from a GPS network. J. Geophys. Res. Solid Earth 2006, 111. [CrossRef]

22. Huang, W.C. Research on Compensation for System Errors of Basic Satellite Products. Ph.D. Thesis, Wuhan University, Wuhan, China, October 2016.

23. Zhao, R.S.; Zhang, G.; Deng, M.J.; Xu, K.; Guo, F.C. Geometric calibration and accuracy verification of the GF-3 satellite. Sensors 2017, 17, 1977. [CrossRef] [PubMed]

24. Jehle, M.; Perler, D.; Small, D.; Schubert, A.; Meier, E. Estimation of Atmospheric Path Delays in TerraSAR-X Data using Models vs. Measurements. J. Sens. 2008, 8, 8479-8491. [CrossRef] [PubMed]

(C) 2020 by the authors. Licensee MDPI, Basel, Switzerland. This article is an open access article distributed under the terms and conditions of the Creative Commons Attribution (CC BY) license (http://creativecommons.org/licenses/by/4.0/). 